\title{
Mucinous Adenocarcinomas Histotype Can Also be a High-Risk Factor for Stage II Colorectal Cancer Patients
}

\author{
Xiang Hu $\mathrm{Hu}^{\mathrm{a}, \mathrm{b}}$ Ya-Qi Li ${ }^{\mathrm{a}, \mathrm{b}}$ Qing-Guo Li ${ }^{\mathrm{a}, \mathrm{b}}$ Yan-Lei Ma ${ }^{\mathrm{a}, \mathrm{b}} \quad$ Jun-Jie Peng ${ }^{\mathrm{a}, \mathrm{b}}$ \\ San-Jun Caia \\ aDepartment of Colorectal Surgery, Fudan University Shanghai Cancer Center, 270 Dong'an Road, \\ Shanghai, bDepartment of Oncology, Shanghai Medical College, Fudan University, Shanghai, China
}

\section{Key Words}

Mucinous adenocarcinoma $\cdot$ Colorectal cancer $\bullet$ Outcome $\cdot$ Poor prognostic features

\begin{abstract}
Background/Aims: Colorectal mucinous adenocarcinoma (MA) has been associated with a worse prognosis than adenocarcinoma (AD) in advanced stages. Little is known about the prognostic impact of a mucinous histotype on the early stages of colorectal cancer with negative lymph node (LN) metastasis. In contrast to the established prognostic factors such as T stage and grading, the histological subtype is not thought to contribute to the therapeutic outcome, although different subtypes can potentially represent different entities. In this study, we aimed to define the prognostic value of mucinous histology in colorectal cancer with negative LNs. Methods: Between 2006 and 2017, a total of 4893 consecutive patients without LN metastasis underwent radical surgery for primary colorectal cancer (MA and AD) in Fudan University Shanghai Cancer Center (FUSCC). Clinical, histopathological, and survival data were analyzed. Results: The incidence of MA was 11\% in 4893 colorectal cancer patients without LN metastasis. The MA patients had a higher T category, a greater percentage of LN harvested, larger tumor size and worse grading than the AD patients ( $p<0.001$ for each). We found that MA histology was correlated with a poor prognosis in terms of relapse in node-negative patients, and MA histology combined with TNM staging may be a feasible method for predicting the relapse rate. Additionally, MA presented as a high-risk factor in patients with negative perineural or vascular invasion and well/moderate-differentiation and showed a more dismal prognosis for stage II patients. Meanwhile, the disease-free survival was identical in MA and $A D$ patients after neo- and adjuvant chemotherapy. Conclusion: MA histology is an independent predictor of poor prognosis due to relapse in LN-negative colorectal cancer patients. Mucinous histology can suggest a possible high risk in early-stage colorectal carcinoma.
\end{abstract}




\section{Cellular Physiology Cell Physiol Biochem 2018;47:630-640 \begin{tabular}{l|l} 
DOI: 10.1159/000490018 & O 2018 The Author(s). Published by S. Karger AG, Basel \\
www.karger.com/cpb
\end{tabular}}

Hu et al.: Mucinous Colorectal Cancer with Negative Node

\section{Introduction}

Colorectal cancer (CRC) is estimated as the third most common cancer and the fourth most frequent cause of cancer-related deaths worldwide [1, 2]. Mucinous adenocarcinoma (MA) is a subset of colorectal cancer, accounting for approximately $10 \%$ of all CRC $[3,4]$. This type of tumor contains neoplastic cells that produce a large amount of extracellular mucin, and the histotype designation requires a more than $50 \%$ mucinous component of the tumor volume [5]. The prognostic outcome of MA patients remains controversial. Some researchers have presented that patients with colorectal MA have a worse prognosis than those with adenocarcinoma (AD) [4, 6, 7], while others have reported no difference [8-11]. The lack of consensus may be attributable to the diversity of patients included (e.g., those with different stages). Most of the previous studies only analyzed and focused on the stage III and IV diseases. Few studies have evaluated the differences in survival between MA and AD for cases of early-stage colorectal cancer with negative node metastasis. Nodal metastases represent a known prognostic factor after surgical resection of CRC. In theory, patients with negative lymph nodes (LNs) have a good outcome, yet a subset of node-negative colorectal cancers still experience an ominous prognosis. The high-risk features in the early stage of colorectal cancer remain valid, coexisting with the $\mathrm{T}$ staging and grading system, although recent efforts have been made to incorporate the histological subtype into a combined risk assessment. The reason why a combined classification system has not been established in the clinical practice may be the ambiguity existing in the prognostic value of the histological subtype in separated stages. Due to the relatively rare occurrence, evaluation of the MA subtype on the outcome of patients is challenging. The purpose of this study was to establish the role of MA in the prognosis for early-stage colorectal carcinoma with negative node metastasis and to identify whether tumor histotype should be taken into account in distinguishing the high-risk features of early-stage colorectal carcinoma.

\section{Materials and Methods}

\section{Patients}

Since 2006, all patients undergoing surgery for colorectal cancer at the Department of colorectal Surgery, Fudan University Shanghai Cancer Center (FUSCC), Shanghai, China, are scheduled for periodic follow-up at our cancer center. All patient data are prospectively entered in the FUSCC database, including age, race, tumor location, year of diagnosis, primary tumor size, histological grade, number of lymph nodes examined, 7th edition TNM stage, preoperative multimodal treatment, details of the surgical procedure, occurrence of complications, postoperative histopathology, application of adjuvant therapy, and followup (date of last visit, tumor recurrence, date of tumor-related or unrelated death, cause-specific and recurrence-free survival). All of the routine preoperative examination showed no distant metastases and no cancer directly invading adjacent organs. Thus, all patients underwent radical surgery with classical lymph node dissection. All specimens were examined by surgeons and pathologists shortly after surgery. The presence of MA, or AD was documented. Classical gland-forming adenocarcinomas with variable size and configuration of the glandular structures were classified as AC. Mucinous adenocarcinoma (MA) is a histological subtype of colorectal cancer in which more than $50 \%$ of the lesion was composed of mucin, typically characterized by pools of extracellular mucin containing malignant epithelium [12]. The status of the lymph node metastasis was evaluated by pathologists using haematoxylin-eosin staining. For this analysis, consecutive complete data sets of patients with resection of colorectal MA and AD with nonmetastatic lymph nodes were extracted between January 2006 and March 2017. Further assessment was performed using the following exclusion criteria: unknown vital status and loss of follow-up data, inadequate surgical data, unspecified tumor location, undetermined staging, patients with a tumor-histology other than MA or AD and patients who died within 30 days after surgery. And then, histological, clinical, and survival data of patients with classical MA were compared with those of patients with AD. All patients from FUSCC dataset have provided written informed consent. The research protocol was reviewed and approved by the institutional review board of the FUSCC. 
Statistical Analysis

Statistical evaluation was performed using IBM SPSS statistics Version 22 (SPSS Inc; IBM Corporation Software Group, Somers, NY). The Chi-square test or Fisher exact test was utilized for exploratory comparisons of patient groups. All statistical tests were performed 2-sided, and P values less than 0.05 were considered to be statistically significant. Observed (unadjusted overall) survival was estimated with the Kaplan-Meier method, and the log-rank (Mantel-Cox) test was used to compare independent subgroups. Cause-specific survival and disease-free survival were used as the primary outcome parameter. Cause-specific survival is equivalent to disease-specific survival regarding the initial malignant disease and considers only tumor related deaths as events [13]. Cox proportional hazard models were used to investigate the effect on survival of multivariable relationships among covariates including the age at diagnosis, gender, stage at diagnosis, histological type, histological grade and treatment. Stage, status of perineural and vascular invasion or any known clinical characteristics supposed to affect the prognosis were the stratified variable. Hazard ratios (HRs) and 95\% confidence intervals (CIs) for multivariate analyses were computed using the Cox proportional hazards regression models.

\section{Results}

\section{Patient characteristics and tumor presentation}

There was a total of 4893 patients with node-negative disease in the FUSCC colorectal cancer dataset from 2006-2017 who met the inclusion criteria for analysis. Among the enrolled patients, 4356 (89\%) adenocarcinomas (ADs) and 537(11\%) mucinous adenocarcinomas (MAs) were observed. A detailed comparison of the relevant characteristics between AD and MA patients is listed in Table 1 . The mean follow-up period was $28.2 \pm 26$ months. At the time of diagnosis, ADs were more often classified as stage I tumors ( $40 \%$ versus $20.3 \%, \mathrm{P}<0.01$ ), while MA presented with advanced tumor categories (stage IIa, $58.4 \%$ versus $40.2 \%$, $\mathrm{P}<0.01$ ). Further, patients with MA were associated with higher T categories (T4: 58.5 vs. $40.2 \%$, $\mathrm{p}<0.001)$ as well as a worse grade of differentiation (G3/G4: 48.1\% vs. 9.8\%, p $<0.001$ ) than patients with AD. In addition, compared with the AD group, the tumor size was larger in the MA group ( $4.9 \pm 2.1 \mathrm{vs.}$ $3.7 \pm 1.7 \mathrm{~cm}, \mathrm{p}<0.001$ ) and accompanied by more lymph node retrieved. Above all, there were no differences in perineural invasion, vascular invasion, age or gender distribution between the two groups. Of note, patients with MA were more likely to receive adjuvant chemotherapy after operation $(50.7 \%$ vs. $40.8 \%$; $\mathrm{P}<0.01)$. Nevertheless, compared to patients with $\mathrm{AD}$, a lower proportion of patients with $\mathrm{MA}$ underwent neo-adjuvant chemotherapy.

Survival (overall survival and diseasefree survival)

Fig. 1A displays the Kaplan-Meier overall survival curves for the different histological subtypes. During the followup, of the 4356 patients with AD, 173 patients (4\%) died, while 22 of the 537

Table 1. Description of the study population between LN-negative MA and $\mathrm{AD}$ colorectal cancer patients. CT indicates chemotherapy; LN, Lymph node; MA, Mucinous adenocarcinomas; AD, adenocarcinomas

\begin{tabular}{|c|c|c|c|}
\hline Variables, n (\%) & $\mathrm{AD}(4356)$ & MA(537) & $\mathrm{P}$ value \\
\hline \multicolumn{4}{|l|}{ Gender } \\
\hline Male & $2642(60.6)$ & $137(25.5)$ & 0.47 \\
\hline Female & $1714(39.4)$ & $220(74.5)$ & \\
\hline Age, years & $60.6 \pm 11.6$ & $59.2 \pm 11.2$ & 0.06 \\
\hline Tumor location & & & $<0.01$ \\
\hline Right side colon & $717(16.9)$ & $192(38.0)$ & \\
\hline Left side colon & $1109(26.2)$ & $115(22.8)$ & \\
\hline Rectum & $2412(56.9)$ & $198(39.2)$ & \\
\hline 7th edition TNM stage & & & $<0.01$ \\
\hline I & $1742(40)$ & $109(20.3)$ & \\
\hline IIa & $862(19.8)$ & $114(21.2)$ & \\
\hline IIb & $1752(40.2)$ & $314(58.5)$ & \\
\hline Grade & & & $<0.01$ \\
\hline well & $366(8.4)$ & $96(17.9)$ & \\
\hline moderate & $3564(81.8)$ & $183(34)$ & \\
\hline poor & $426(9.8)$ & $258(48.1)$ & \\
\hline Tumor stage & & & $<0.01$ \\
\hline $\mathrm{T} 1$ & $601(13.8)$ & $9(1.7)$ & \\
\hline $\mathrm{T} 2$ & $1141(26.2)$ & $100(18.6)$ & \\
\hline T3 & $862(19.8)$ & $114(21.2)$ & \\
\hline $\mathrm{T} 4$ & $1752(40.2)$ & $314(58.5)$ & \\
\hline Tumor size $(\mathrm{cm})$ & & & $<0.01$ \\
\hline Mean & $3.7 \pm 1.7$ & $4.9 \pm 2.1$ & \\
\hline Lymph node examined & & & 0.001 \\
\hline Median & $16 \pm 7$ & $19 \pm 8$ & \\
\hline Perineural invasion & & & 0.1 \\
\hline Negative & $3919(89.9)$ & $495(92.1)$ & \\
\hline Positive & $437(10.1)$ & $42(7.9)$ & \\
\hline Vascular invasion & & & 0.68 \\
\hline Negative & $4060(93.2)$ & $503(93.6)$ & \\
\hline Positive & $296(6.8)$ & $34(6.4)$ & \\
\hline Adjuvant Chemotherapy & & & $<0.01$ \\
\hline No & $2579(59.2)$ & $265(49.3)$ & \\
\hline Yes & $1777(40.8)$ & $272(50.7)$ & \\
\hline Neo-adjuvant CT & & & $<0.01$ \\
\hline No & $4024(92.4)$ & $520(96.84)$ & \\
\hline Yes & $332(7.6)$ & $17(3.16)$ & \\
\hline
\end{tabular}


patients with MA $(4.1 \%)$ died. There was no significant difference in the overall survival between patients with $\mathrm{AD}$ and patients with $\mathrm{MA}$ histology ( $\mathrm{P}=0.597)$. Notably, a divergence in the overall survival seemed to emerge from the Kaplan-Meier curves, implying much worse prognosis of MA, which was when adjuvant chemotherapy was not used $(\mathrm{P}=0.019$; Fig. 1B). However, when comparing $\mathrm{MA}$ and $\mathrm{AD}$ patients who all received adjuvant chemotherapy, we did not observe any significant difference in survival ( $\mathrm{P}=0.071$; Fig. $1 \mathrm{C}$ ). Furthermore, pairwise comparisons of data stratified into the categories of tumor grade, retrieved LNs, perineural invasion, vascular invasion, tumor location and the status of neo-adjuvant chemotherapy did not show differences between the two groups (Table 2).

We further compared the differences in disease-free survival rates between the MA and AD groups. The Kaplan-Meier curves for diseasefree survival are presented in Fig. 2. Of all the 4893 nodenegative colorectal patients, 441 had a local relapse or distant metastasis. The 5-year DFS of the AD patients was $83.3 \%$, while that of the MA patients was $75 \%$, indicating a worse prognosis for the MA group. To identify the mucinousrelated prognostic factors contributing to the DFS, all patients with positive or negative perineural invasion, vascular invasion or any known clinical characteristics and their long-term follow-up data were analyzed. Fig. 3 depicts the disease-free survival curves for colorectal MA and AD patients across a 10-year span of follow-up. Differences were found in survival between MA and AD in patients with negative perineural and vascular invasion. The estimated mean disease-free survival of MA patients was significantly lower than that of $\mathrm{AD}$ patients with no perineural (86 vs. 165 months; $\mathrm{P}<0.001$ ) or vascular invasion (86 vs. 160 months; $\mathrm{P}=0.002$ ). However, there were no differences in disease-free survival between MA and AD patients with positive perineural or vascular invasion $(\mathrm{P}=0.598$ and $\mathrm{P}=$ 0.914 , respectively).

T stage accounts for DFS differences in different histological types. Differences in the DFS between patients with AD and those with MA histology were found in stage II colorectal
Table 2. Pairwise Comparisons in stratification between LN-negative MA and $\mathrm{AD}$ colorectal cancer patients. CT indicates chemotherapy; MA, Mucinous adenocarcinomas; AD, adenocarcinomas

\begin{tabular}{lcc}
\hline Variables & Log-Rank $\mathrm{x}^{2}$ test & $\mathrm{P}$ value \\
\hline 7th edition TNM stage & & \\
I & 0.685 & 0.408 \\
II & 1.754 & 0.185 \\
Tumor stages & & \\
T1 & 0.225 & 0.635 \\
T2 & 0.429 & 0.512 \\
T3 & 0.245 & 0.620 \\
T4 & 1.084 & 0.298 \\
Perineural invasion & & \\
Negative & 0.021 & 0.884 \\
Positive & 0.640 & 0.424 \\
Vascular invasion & & \\
Negative & 0.072 & 0.789 \\
Positive & $<0.01$ & 0.997 \\
Neo-adjuvant CT & & \\
No & 0.020 & 0.886 \\
Yes & 0.101 & 0.751 \\
Tumor location & & \\
colon & & \\
rectum & 1.180 & 0.277 \\
& 0.271 & 0.602 \\
\hline
\end{tabular}




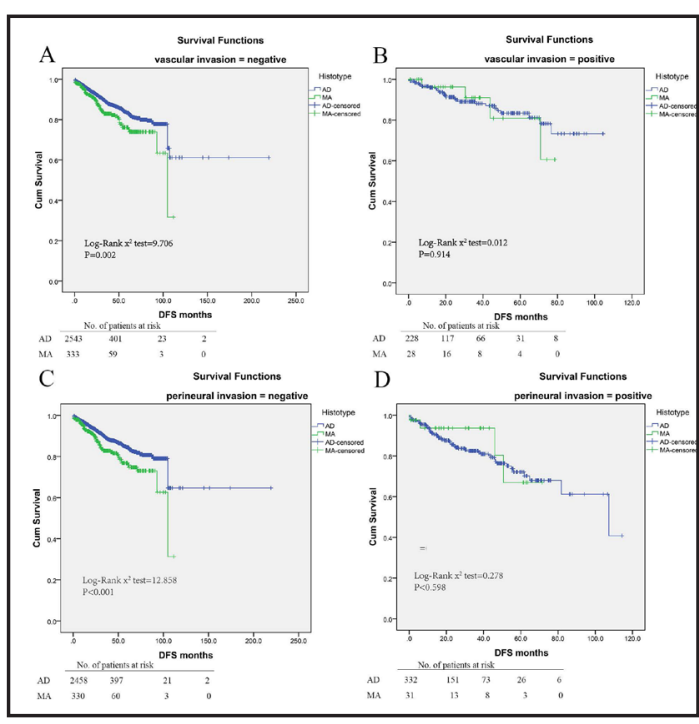

Fig. 3. Kaplan-Meier survival curves comparing cancer disease free survival in Mucinous adenocarcinomas (MA) and Adenocarcinomas(AD) of colorectal cancer are shown for (A)tumors with no vascular invasion, (B)tumors with positive vascular invasion, (C)tumors with no perineural invasion, (D)tumors with positive perineural invasion.

cancer and in T4 diseases but not in stage I cancers or other $\mathrm{T}$ stages (Fig. 4). With a mean disease-free survival of 85 months, the prognosis of MA cancers was significantly worse than that of $\mathrm{AD}$ (mean disease-free survival 148 months, $P=0.0024$ ). Stratified by $\mathrm{T}$ stage, the mucinous subtype had an obvious, negative independent influence on prognosis in the T4 group (82 vs. 140 months; $\mathrm{P}=0.045$ ). However, no significant difference of metastasis or relapse was observed between MA and AD patients in stage I or other T stages.

Similarly, differences in the DFS between MA and AD cancers were found in patients without adjuvant chemotherapy and in those who did not undergo neo-adjuvant chemoradiotherapy. Patients with the MA histotype (Fig. 5) had a worse diseasefree survival than the AD histotype patients when they were not treated with neo- or adjuvant chemotherapy (86 vs. 161 months, $P$ $<0.001$; and 86 vs. 128 months, $\mathrm{P}<0.001$; respectively). However, for patients who underwent neo- and adjuvant chemotherapy, the differences between patients with MA and AD were not of statistical significance $(\mathrm{P}=0.418$ and $\mathrm{P}=0.347)$.

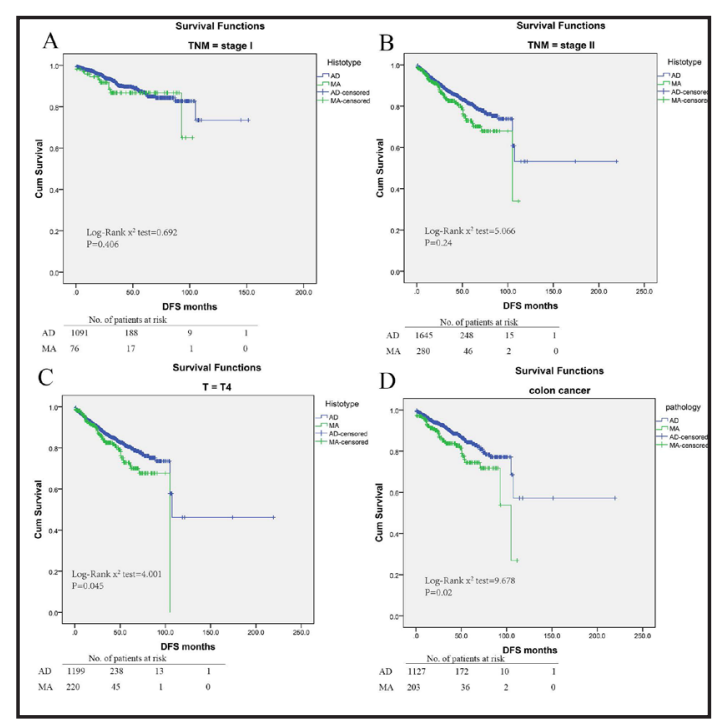

Fig. 4. Kaplan-Meier survival curves comparing cancer disease free survival in Mucinous adenocarcinomas (MA) and Adenocarcinomas(AD) of colorectal cancer are shown for (A)stage I tumors, (B)stage II tumors, (C)stage T4 tumors and (D)tumor location.

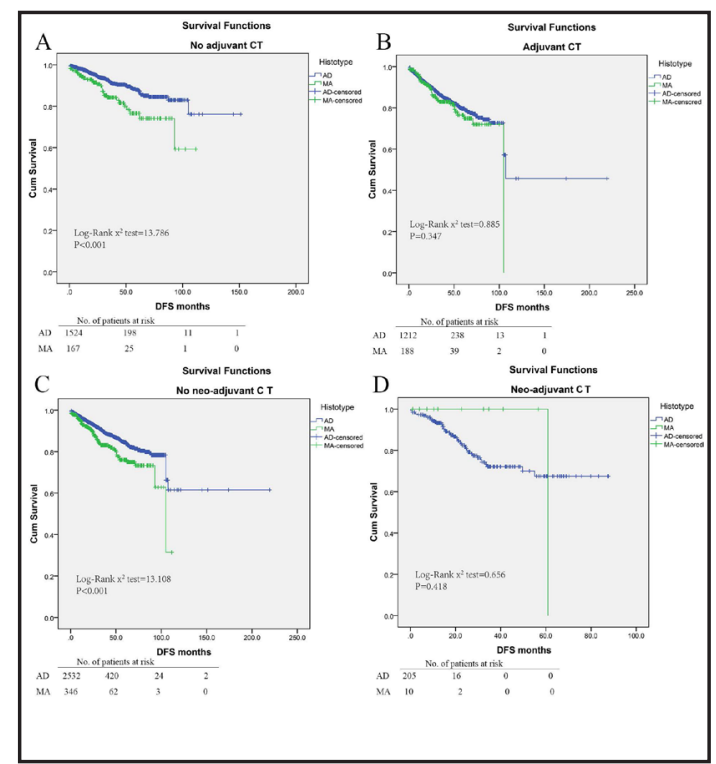

Fig. 5. Kaplan-Meier survival curves comparing cancer disease free survival in Mucinous adenocarcinomas (MA) and Adenocarcinomas(AD) of colorectal cancer are shown for (A) patients with no adjuvant chemotherapy(CT), (B) patients with adjuvant chemotherapy(CT), (C) patients with no Neo-adjuvant chemotherapy(CT), (D) patients with Neo-adjuvant chemotherapy(CT). 
Fig. 6. Kaplan-Meier survival curves comparing cancer disease free survival in Mucinous adenocarcinomas (MA) and Adenocarcinomas(AD) of colorectal cancer are shown for (A) tumors with well and moderate grade, (B) tumors with poor grade differentiation.

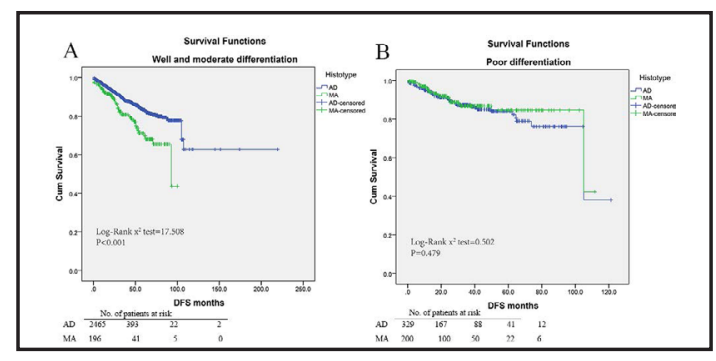

In recent years, histological grading of early colorectal cancers has played a crucial role in estimating prognosis. Thus, subdividing colon and rectal cancers into two groups, well/ moderate-differentiated tumors as a low-risk group and poorly differentiated tumors as a highrisk group, was conducted for prognosis estimation. Based on the well/moderate-differentiated grading, the disease-free survival of patients with MA was much lower than that of patients with $\mathrm{AD}$ (76 vs. 162 months; $\mathrm{P}<$ 0.001 ), while the prognoses of $\mathrm{MA}$ and $\mathrm{AD}$ were nearly identical for colorectal cancer in the poor differentiation group $(\mathrm{P}=0.479$; Fig. 6).

Finally, stratified by tumor location, the mucinous subtype had an obvious, negative independent influence on

Table 3. Univariate and Multivariate analyses of prognostic factors for cancer specific survival CT indicates chemotherapy; NI, NOT included; MA, Mucinous adenocarcinomas; AD, adenocarcinomas; CT, ChemotherapyMA, Mucinous adenocarcinomas; $\mathrm{AD}$, adenocarcinomas

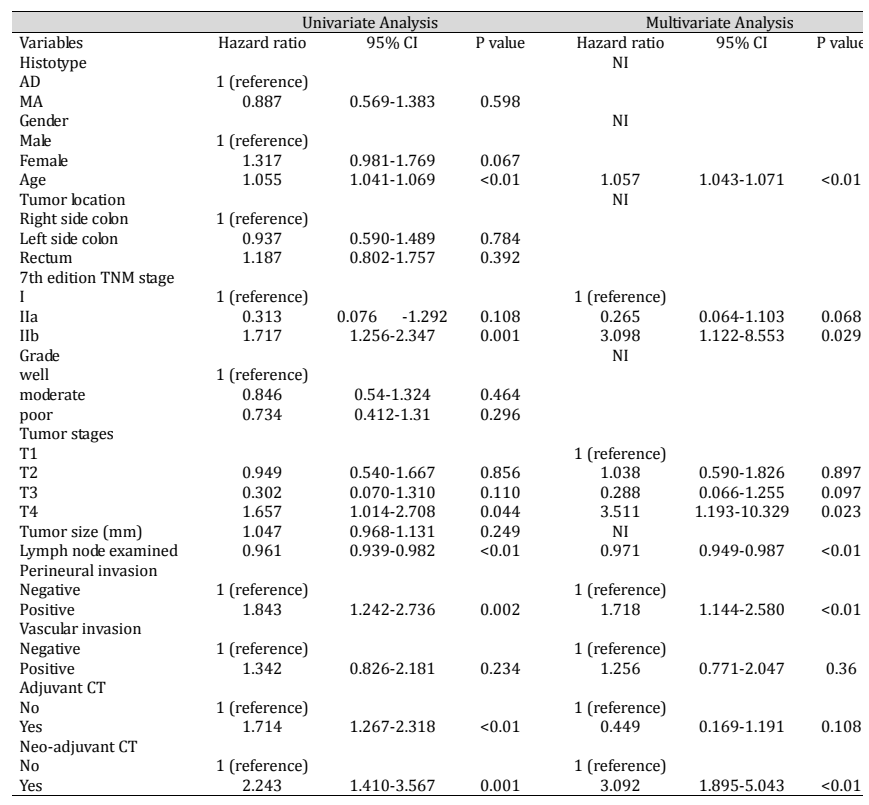
prognosis in colon cancers (84 vs. 156 months, $\mathrm{P}=0.02$; Fig. 4D), while a statistical significance was not observed in the rectal cancer group ( $\mathrm{p}=0.117)$.

\section{Predictors of mortality and relapse rate}

Unadjusted associations with mortality are displayed in Table 3. The mucinous type (univariate Cox HR, 0.887; 95\% CI, 0.569-1.383; P = 0.598), female gender (HR, 1.317; 95\% CI, 0.981-1.769; $\mathrm{P}=0.067$ ), tumor grade (HR, 0.734; 95\% CI, 0.412-1.31; $\mathrm{P}=0.296$ ), tumor size (HR, 1.047; 95\% CI, 0.968-1.131; P = 0.249) and vascular invasion (HR, 1.342; 95\% CI, $0.826-2.181 ; \mathrm{P}=0.234$ ) added no additional risk as mortality risk factors.

Factors associated with increased mortality included age at diagnosis (HR, 1.055; 95\% CI, 1.041-1.069; $\mathrm{P}<0.001$ ), TNM stage (HR, 1.717; 95\% CI, 1.256-2.347; $\mathrm{P}=0.001)$, T stage (HR, 1.657; 95\% CI, 1.014-2.708; $\mathrm{P}=0.044)$, the number of retrieved LNs $(\mathrm{HR}, 1.961 ; 95 \%$ CI, 0.939-0.982; $\mathrm{P}<0.001$ ), perineural invasion (HR, 1.843; 95\% CI, 1.242-2.736; P = 0.002), and the status of chemotherapy. Further multivariate analysis showed that age, TNM stage, perineural invasion, and neo-adjuvant chemotherapy were independent prognostic factors in colorectal carcinoma with negative node metastasis.

The results of the Cox proportional hazards analysis for factors associated with diseasefree survival in node-negative disease are summarized in Table 4. MA histology (multivariate 
Cox HR, 1.506; 95\% CI, 1.146-1.979; $\mathrm{P}=0.003)$, age (multivariate Cox HR, 1.024; 95\% CI, 1.015-1.032; $\mathrm{P}<0.001)$, TNM stage (HR, 2.733; 95\% CI, 1.431-5.222; $\mathrm{P}=0.002), \mathrm{T}$ stage $(\mathrm{HR}$, 3.094; 95\% CI, 1.534-6.242; $\mathrm{P}=0.0002)$, the number of retrieved LNs (HR, 0.971; 95\% CI, 0.956-0.987; $\mathrm{P}<$ 0.001 ), perineural invasion (HR, 1.707; 95\% CI, 1.3012.238; $\mathrm{P}=0.001$ ), and neo-adjuvant CRT were significantly associated with disease-free survival. Moreover, multivariate Cox regression survival analysis adjusted for mucinous histology, age, TNM stage IIa, T4, retrieved LNs, perineural invasion and neo-adjuvant chemotherapy consistently validated the strong correlations with disease-free survival in node-negative patients, as depicted in Fig. 7.
Table 4. Univariate and Multivariate analyses of prognostic factors for cancer disease-free survival. CT indicates chemotherapy; NI, NOT included; MA, Mucinous adenocarcinomas; AD, adenocarcinomas; CT, Chemotherapyincluded; MA, Mucinous adenocarcinomas; AD, adenocarcinomas; CT, Chemotherapy

\begin{tabular}{|c|c|c|c|c|c|c|}
\hline \multirow[b]{2}{*}{ Variables } & \multicolumn{3}{|c|}{ Univariate Analysis } & \multicolumn{3}{|c|}{ Multivariate Analysis } \\
\hline & Hazard ratio & $95 \% \mathrm{CI}$ & $P$ value & Hazard ratio & $95 \% \mathrm{CI}$ & P value \\
\hline Histotype & & & & & & \\
\hline $\mathrm{AD}$ & 1 (reference) & & & 1 (reference) & & \\
\hline $\begin{array}{l}\text { MA } \\
\text { Gender }\end{array}$ & 1.472 & $1.144-1.896$ & 0.003 & $\begin{array}{c}1.506 \\
\mathrm{NI}\end{array}$ & $1.146-1.979$ & 0.003 \\
\hline Male & 1 (reference) & & & & & \\
\hline Female & 1.158 & $0.955-1.404$ & 0.137 & & & \\
\hline Age & 1.024 & $1.015-1.032$ & $<0.01$ & 1.024 & $1.015-1.032$ & $<0.01$ \\
\hline Tumor location & & & & NI & & \\
\hline Right side colon & 1 (reference) & & & & & \\
\hline Left side colon & 1.114 & $0.829-1.498$ & 0.474 & & & \\
\hline Rectum & 1.123 & $0.864-1.459$ & 0.386 & & & \\
\hline 7th edition TNM stage & & & & & & \\
\hline I & 1 (reference) & & & 1 (reference) & & \\
\hline IIa & 1.165 & $0.752-1.806$ & 0.494 & 1.067 & $0.674-1.689$ & 0.783 \\
\hline IIb & 1.721 & $1.393-2.126$ & $<0.01$ & 2.733 & $1.431-5.222$ & 0.002 \\
\hline Grade & & & & NI & & \\
\hline well & 1 (reference) & & & & & \\
\hline moderate & 0.882 & $0.648-1.202$ & 0.427 & & & \\
\hline poor & 0.970 & $0.664-1.415$ & 0.873 & & & \\
\hline Tumor stage & & & & & & \\
\hline T1 & 1 (reference) & & & 1 (reference) & & \\
\hline T2 & 1.040 & $0.709-1.526$ & 0.841 & 1.132 & $0.770-1.666$ & 0.528 \\
\hline T3 & 1.197 & $0.718-1.995$ & 0.491 & 1.208 & $0.717-2.035$ & 0.478 \\
\hline $\mathrm{T} 4$ & 1.768 & $1.261-2.479$ & 0.001 & 3.094 & $1.534-6.242$ & 0.002 \\
\hline Tumor size (mm) & 0.996 & $0.945-1.051$ & 0.895 & NI & & \\
\hline $\begin{array}{l}\text { Lymph node examined } \\
\text { Perineural invasion }\end{array}$ & 0.970 & $0.956-0.984$ & $<0.01$ & 0.971 & $0.956-0.987$ & $<0.01$ \\
\hline Negative & 1 (reference) & & & 1 (reference) & & \\
\hline Positive & 1.815 & $1.392-2.367$ & $<0.01$ & 1.707 & $1.301-2.238$ & $<0.01$ \\
\hline Vascular invasion & & & & NI & & \\
\hline Negative & 1 (reference) & & & & & \\
\hline Positive & 1.054 & $0.735-1.511$ & 0.774 & & & \\
\hline Adjuvant CT & & & & & & \\
\hline No & 1 (reference) & & & 1 (reference) & & \\
\hline Yes & 1.564 & $1.286-1.902$ & $<0.01$ & 0.581 & $0.314-1.076$ & 0.084 \\
\hline Neo-adjuvant CT & & & & & & \\
\hline No & 1 (reference) & & & 1 (reference) & & \\
\hline Yes & 2.199 & $1.627-2.972$ & $<0.01$ & 2.251 & $1.642-3.086$ & $<0.01$ \\
\hline
\end{tabular}

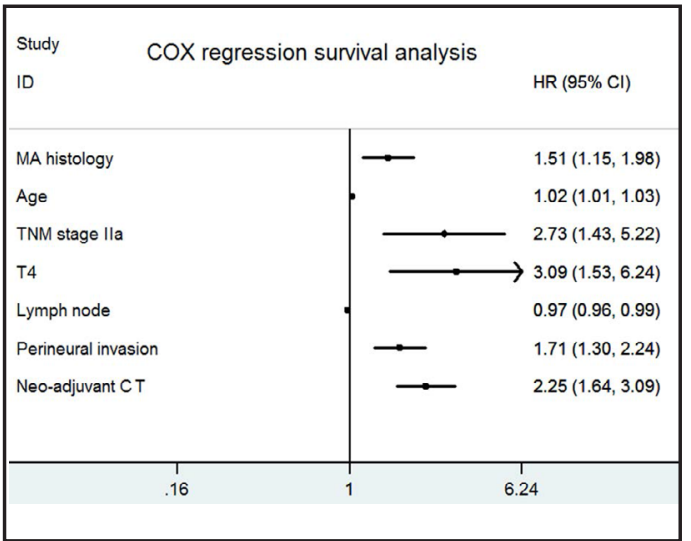

Fig. 7. Different factors were analyzed for their association with disease free survival using the Cox regression model. The hazard ratio and 95\% confidence interval (CI) are plotted for each factor. CT indicates chemotherapy; MA, Mucinous adenocarcinomas.

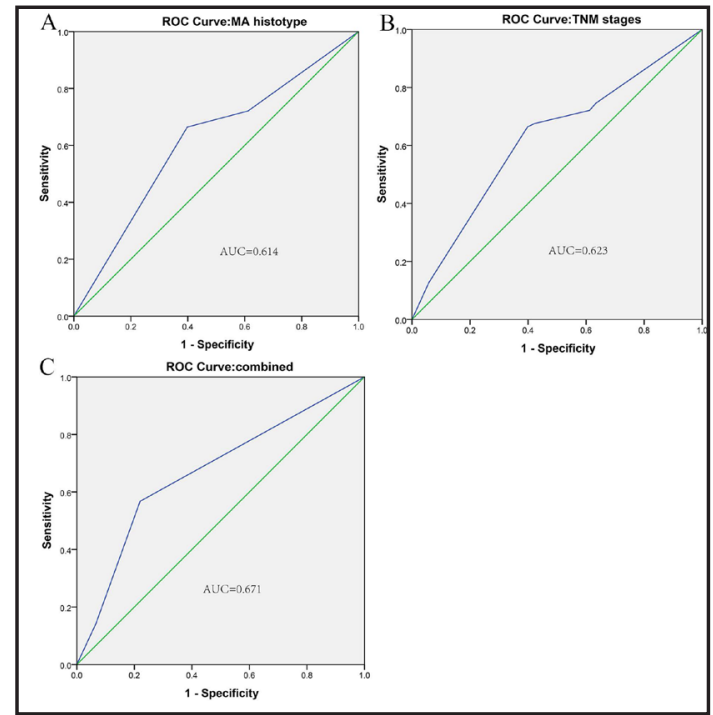

Fig. 8. The receiver operating characteristic (ROC) curves for predicting patient disease free survival using the TNM classification (B), Mucinous Adenocarcinomas (MA) histology (A) or a combination of the two factors (C). The area under the curve (AUC) are shown in the plots. 
Finally, the receiver operating characteristic curves were determined for the prediction of disease-free survival in node-negative colorectal patients using the TNM stage, mucinous histology, or a combination of both (Fig. 8). The area under the curve (AUC) that was predicted based on TNM staging (0.623) was higher than that for the MA histology-based model $(0.614)$, and the combination of both factors achieved the highest AUC value (0.671).

\section{Discussion}

In this study, the effect of mucinous histotype in node-negative colorectal cancer on survival and tumor presentation was analyzed. First, patients with MA have worse relapse and survival rates, and mucinous histology was not an independent prognostic factor for overall survival in node-negative colorectal cancer patients. In addition, another novel finding is that mucinous histology is an independent prognostic factor in low-risk cases of early-stage colorectal cancer. The mucinous histotype posed an obvious negative influence on DFS in stage II cancer, as well as well/moderate-differentiated tumors, when no vascular or perineural invasion existed.

In recent years, the subset of node-negative colorectal cancer patients who still experienced a poor prognosis has been a topic of considerable research. Hence, several specific high-risk features are recommended by the American Society of Clinical Oncology guidelines, The National Comprehensive Cancer Network and the European Society for Medical Oncology [14-16], including perforation, T4 tumor, suboptimal lymph node sampling, and poor differentiation, as well as colonic obstruction and lymphovascular or perineural invasion. However, these clinical and pathological factors do not reliably predict the realworld prognosis. A partial component of the reason can be explained by the incompetent high-risk stratification. To date, mucinous histology has not been considered as a high-risk feature, and it has not been considered in the evaluation of adjuvant chemotherapy trials for the early-stage disease. In the present study, we observed an obvious negative prognostic role of MA for patients with node-negative colorectal cancers. The prognosis of MA, with a 5 -year disease-free survival of $75 \%$, was significantly worse than that of AD, with a 5-year disease-free survival of $83.3 \%(P=0.003)$. To explain this, a specific histopathological and recurrent pattern of MA from $A D$ has been suggested in this study and others $[17,18]$. For instance, MA presented with advanced tumor categories more often than AD (stage IIa, $58.4 \%$ versus $40.2 \%, \mathrm{P}<0.01$ ). Further, compared to patients with $\mathrm{AD}$, those with MA were associated with higher T categories (T4: 58.5 vs. $40.2 \%, \mathrm{p}<0.001$ ), as well as a worse grade of differentiation (G3/G4: 48.1\% vs. 9.8\%, p < 0.001). In addition, when compared to AD, tumors classified as large size were more common in MA ( $4.9 \pm 2.1 \mathrm{vs.} 3.7 \pm 1.7 \mathrm{~cm}, \mathrm{p}<0.001)$. Above all, research on molecular variations might help to identify MA patients with a higher risk for malignant tumor behavior in terms of survival. For example, in comparison with $\mathrm{AD}$, MA has microsatellite instability more frequently, indicating an alternative oncogenic pathway contributing to the disease [19]. Whenever microsatellite stability was observed, however, a notably reduced rate of copy-number aberrations was characterized in MA when compared to that in $\mathrm{AD}[20]$. In addition, active BRAF mutations were more frequently found in patients with MA and were associated with an infiltrative pattern of tumor growth [18, 19]. In the end, loss of the p53 gene, which plays a vital role in regulating the cell cycle, led to uncontrolled tissue growth and aggressive tumors. In accordance, the expression of the p53 protein was lower in MA [21]. In all, these molecular variations of MA that result in clinical features might explain the worse prognosis.

However, poor prognosis for MA has not always been consistently observed [18, 22, 23]. Several studies reported that large mucinous areas did not have independent prognostic value in a multivariate analysis. This can be attributed to the fact that the majority of these reports only presented overall survival rather than disease-free survival in their analyses. In fact, there are no contradictions in the prognostic value of mucinous histology. As demonstrated by Nitsche U [9], mucinous histology was not an independent prognostic factor for overall 
survival; however, MA was associated with significant differences regarding the cumulative incidence of locoregional and distant recurrence. In line with our study, a divergence in the overall survival was not demonstrated by the Kaplan-Meier curves, yet the 5-year diseasefree survival of MA patients was significantly worse compared with that of AD patients $(75 \%$ VS $83.3 \%, P=0.003$ ). Given that tumor behavior is routinely evaluated by disease recurrence and metastasis, DFS is one of the most sensitive factors of the biological characteristics. Unlike overall survival, DFS is less influenced by disparities in the treatment of relapsed disease, the management of comorbidities, or the differential rates of death from competing causes irrespective to cancer. Furthermore, the cancer stages were not taken into consideration. Patients with MA had worse survival than patients with AD in stage III diseases, while no such difference was observed in patients with stage IV [17, 24]. Therefore, the heterogeneity of stages may explain some of the contradictions. To our knowledge, we first reported the largest dataset of patients with MA and AD in early-stage node-negative colorectal carcinoma and identified the role of the mucinous histotype in early-stage colorectal cancer. In addition, this study is strengthened by the considerably large number of patients and our thoroughly documented clinicopathological and long-term follow-up data.

It is interesting to note that MA has a close relationship with worse outcomes among patients with low-risk stage II cancer who are negative for perineural and vascular invasion or well/moderate-differentiated grading. This observation could be a reflection of mucinous histology as high-risk feature in colorectal cancers. The negative prognostic effect of MA becomes more readily apparent when the adverse effects are minimal in the low-risk group. In accordance with the molecular background, low expression of the adhesion molecules, such as E-cadherin and $\beta$-catenin may result in reduced cell adhesion in regions of high mucinous content and may promote scattering of cancer cells, further leading to local recurrence, distant metastasis and poor prognosis $[25,26]$. These findings are supported by another cohort [27] in which a preponderance of local relapse was observed. In particular, local recurrence may be increased in areas of high extracellular mucus content. Fortunately, in this setting, a good response was observed in MA patients with the addition of chemotherapy. Neo- and adjuvant chemotherapy for MA in stage II cancer are associated with an improved DFS. Patients with the MA histotype had a worse disease-free survival than AD patients without neo- and adjuvant chemotherapy (86 vs. 161 months, $\mathrm{P}<0.001$; and 86 vs. 128 months, $\mathrm{P}<0.001$; respectively); however, when neo- and adjuvant chemotherapy were added, the differences in DFS between MA and AD were not of statistical significance. In agreement with our study [10], a recent analysis suggested that a comparable benefit was derived from adjuvant chemotherapy for both stage II and III MA and AD after radical resection. In contrast, a poor response to chemotherapy was previously demonstrated in MA patients who received palliative chemotherapeutic treatment $[24,28,29]$. The explanations behind the discrepancy may be due to the adjuvant and palliative settings. As MA seems clearly responsive to adjuvant chemotherapy, it is reasonable to postulate that the data from palliative treatment should not be extrapolated to the adjuvant setting.

Several additional limitations are important to consider in interpreting the results of the present study. We were not able to compare the prognosis regarding the amount of mucus. In addition, decisions regarding neo- and adjuvant treatments are invariably associated several personal, surgical, and physician-associated factors, all of which were beyond the scope of information available. In particular, we were unable to account for confounding factors, such as comorbidities and physical status. Thus, selection bias likely informed many of the adjuvant treatment decisions.

In conclusion, no statistically significant differences in overall survival were found between patients with MA and AD colorectal carcinoma with negative node. However, when analyzing the relapse rate, patients with MA had a much worse disease-free survival than patients with $\mathrm{AD}$ for stage II diseases. Above all, grading differentiation and perineural and vascular invasion status contributed useful discriminatory information for MA concerning the DFS within stage II cancers. MA presents worse outcomes among patients with low-risk stage II cancer who are negative for perineural and vascular invasion or well/moderate-

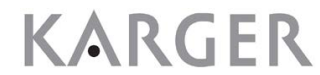


differentiated grading. However, MA seems clearly responsive to chemoradiotherapy, since patients treated with neo- and adjuvant chemotherapy showed no significant differences between the two subtypes of CRC. In all, the results of our study are currently the best evidence showing that MA histology is an independent predictor of poor prognosis due to the frequency of relapse in LN-negative colorectal cancer patients.

\section{Acknowledgements}

This work was supported by the National Natural Science Foundation of China (Grant No. 81672374).

\section{Disclosure Statement}

No conflict of interests exists.

\section{References}

1 Torre LA, Bray F, Siegel RL, Ferlay J, Lortet-Tieulent J, Jemal A: Global cancer statistics, 2012. CA Cancer J Clin 2015;65:87-108.

- Weitz J, Koch M, Debus J, Hohler T, Galle PR, Buchler MW: Colorectal cancer. Lancet 2005;365:153-165.

3 Consorti F, Lorenzotti A, Midiri G, Di Paola M: Prognostic significance of mucinous carcinoma of colon and rectum: a prospective case-control study. J Surg Oncol 2000;73:70-74.

4 Green JB, Timmcke AE, Mitchell WT, Hicks TC, Gathright JB, Jr., Ray JE: Mucinous carcinoma--just another colon cancer? Dis Colon Rectum 1993;36:49-54.

5 Hamilton SR, Aaltonen LA. Pathology and genetics: tumours of the digestive system. In Hamilton SR, Aaltonen LA (eds), World Health Organization Classification of Tumours, 3rd edition. Lyon, France: IARC 2000; 103-143.,

6 Nozoe T, Anai H, Nasu S, Sugimachi K: Clinicopathological characteristics of mucinous carcinoma of the colon and rectum. J Surg Oncol 2000;75:103-107.

7 Kanemitsu Y, Kato T, Hirai T, Yasui K, Morimoto T, Shimizu Y, Kodera Y, Yamamura Y: Survival after curative resection for mucinous adenocarcinoma of the colorectum. Dis Colon Rectum 2003;46:160-167.

$>8$ Purdie CA, Piris J: Histopathological grade, mucinous differentiation and DNA ploidy in relation to prognosis in colorectal carcinoma. Histopathology 2000;36:121-126.

-9 Nitsche U, Friess H, Agha A, Angele M, Eckel R, Heitland W, Jauch KW, Krenz D, Nussler NC, Rau HG, Ruppert R, Schubert-Fritschle G, Wilhelm D, Werner J, Engel J: Prognosis of mucinous and signet-ring cell colorectal cancer in a population-based cohort. J Cancer Res Clin Oncol 2016;142:2357-2366.

10 Catalano V, Loupakis F, Graziano F, Bisonni R, Torresi U, Vincenzi B, Mari D, Giordani P, Alessandroni P, Salvatore L, Fornaro L, Santini D, Baldelli AM, Rossi D, Giustini L, Silva RR, Falcone A, D’Emidio S, Rocchi M, Luzi Fedeli S: Prognosis of mucinous histology for patients with radically resected stage II and III colon cancer. Ann Oncol 2012;23:135-141.

-11 Yarbro JW, Page DL, Fielding LP, Partridge EE, Murphy GP: American Joint Committee on Cancer prognostic factors consensus conference. Cancer 1999;86:2436-2446.

12 Bosman FT CF, Hruban RH, Theise ND: WHO classificationof tumours of the digestive system, 4th edn. WorldHealth Organization, International Agency for Research on Cancer,Geneva. 2010

13 Nitsche U, Maak M, Schuster T, Kunzli B, Langer R, Slotta-Huspenina J, Janssen KP, Friess H, Rosenberg R: Prediction of prognosis is not improved by the seventh and latest edition of the TNM classification for colorectal cancer in a single-center collective. Ann Surg 2011;254:793-800; discussion 800-791.

14 Benson AB, 3rd, Schrag D, Somerfield MR, Cohen AM, Figueredo AT, Flynn PJ, Krzyzanowska MK, Maroun J, McAllister P, Van Cutsem E, Brouwers M, Charette M, Haller DG: American Society of Clinical Oncology recommendations on adjuvant chemotherapy for stage II colon cancer. J Clin Oncol 2004;22:3408-3419. 


\section{Cellular Physiology Cell Physiol Biochem 2018;47:630-640

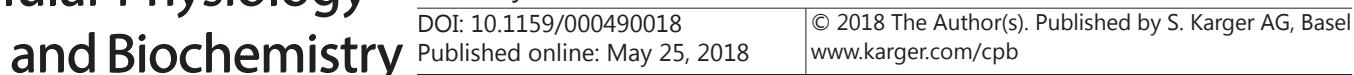

Hu et al.: Mucinous Colorectal Cancer with Negative Node

15 National Comprehensive Cancer Network Guidelines. nccn.org/professionals/physician_gls/f_guidelines. asp. Accessed May 15, 2016.

-16 Schmoll HJ, Van Cutsem E, Stein A, Valentini V, Glimelius B, Haustermans K, Nordlinger B, van de Velde CJ, Balmana J, Regula J, Nagtegaal ID, Beets-Tan RG, Arnold D, Ciardiello F, Hoff P, Kerr D, Kohne CH, Labianca R, Price T, Scheithauer W, Sobrero A, Tabernero J, Aderka D, Barroso S, Bodoky G, Douillard JY, El Ghazaly H, Gallardo J, Garin A, Glynne-Jones R, Jordan K, Meshcheryakov A, Papamichail D, Pfeiffer P, Souglakos I, Turhal S, Cervantes A: ESMO Consensus Guidelines for management of patients with colon and rectal cancer. a personalized approach to clinical decision making. Ann Oncol 2012;23:2479-2516.

$\checkmark 17$ Hugen N, Verhoeven RH, Radema SA, de Hingh IH, Pruijt JF, Nagtegaal ID, Lemmens VE, de Wilt JH: Prognosis and value of adjuvant chemotherapy in stage III mucinous colorectal carcinoma. Ann Oncol 2013;24:2819-2824.

18 Hyngstrom JR, Hu CY, Xing Y, You YN, Feig BW, Skibber JM, Rodriguez-Bigas MA, Cormier JN, Chang GJ: Clinicopathology and outcomes for mucinous and signet ring colorectal adenocarcinoma: analysis from the National Cancer Data Base. Ann Surg Oncol 2012;19:2814-2821.

19 Hugen N, Simons M, Halilovic A, van der Post RS, Bogers AJ, Marijnissen-van Zanten MA, de Wilt JH, Nagtegaal ID: The molecular background of mucinous carcinoma beyond MUC2. J Pathol Clin Res 2015;1:317.

20 Hugen N, Simmer F, Mekenkamp LJ, Koopman M, van den Broek E, de Wilt JH, Punt CJ, Ylstra B, Meijer GA, Nagtegaal ID: Reduced rate of copy number aberrations in mucinous colorectal carcinoma. Oncotarget 2015;6:25715-25725.

21 Hanski C, Tiecke F, Hummel M, Hanski ML, Ogorek D, Rolfs A, Schmitt-Graff A, Stein H, Riecken EO: Low frequency of p53 gene mutation and protein expression in mucinous colorectal carcinomas. Cancer Lett 1996;103:163-170.

-22 Verhulst J, Ferdinande L, Demetter P, Ceelen W: Mucinous subtype as prognostic factor in colorectal cancer: a systematic review and meta-analysis. J Clin Pathol 2012;65:381-388.

23 Xie L, Villeneuve PJ, Shaw A: Survival of patients diagnosed with either colorectal mucinous or nonmucinous adenocarcinoma: a population-based study in Canada. Int J Oncol 2009;34:1109-1115.

-24 Mekenkamp LJ, Heesterbeek KJ, Koopman M, Tol J, Teerenstra S, Venderbosch S, Punt CJ, Nagtegaal ID: Mucinous adenocarcinomas: poor prognosis in metastatic colorectal cancer. Eur J Cancer 2012;48:501-509.

-25 Borger ME, Gosens MJ, Jeuken JW, van Kempen LC, van de Velde CJ, van Krieken JH, Nagtegaal ID: Signet ring cell differentiation in mucinous colorectal carcinoma. J Pathol 2007;212:278-286.

26 Sung CO, Seo JW, Kim KM, Do IG, Kim SW, Park CK: Clinical significance of signet-ring cells in colorectal mucinous adenocarcinoma. Mod Pathol 2008;21:1533-1541.

27 Nitsche U, Zimmermann A, Spath C, Muller T, Maak M, Schuster T, Slotta-Huspenina J, Kaser SA, Michalski CW, Janssen KP, Friess H, Rosenberg R, Bader FG: Mucinous and signet-ring cell colorectal cancers differ from classical adenocarcinomas in tumor biology and prognosis. Ann Surg 2013;258:775-782; discussion 782-773.

28 Catalano V, Loupakis F, Graziano F, Torresi U, Bisonni R, Mari D, Fornaro L, Baldelli AM, Giordani P, Rossi D, Alessandroni P, Giustini L, Silva RR, Falcone A, D’Emidio S, Fedeli SL: Mucinous histology predicts for poor response rate and overall survival of patients with colorectal cancer and treated with first-line oxaliplatinand/or irinotecan-based chemotherapy. Br J Cancer 2009;100:881-887.

29 Negri FV, Wotherspoon A, Cunningham D, Norman AR, Chong G, Ross PJ: Mucinous histology predicts for reduced fluorouracil responsiveness and survival in advanced colorectal cancer. Ann Oncol 2005;16:13051310 . 